\title{
An unidentified variable gamma-ray source near the galactic plane detected by COMPTEL
}

\author{
S. Zhang ${ }^{1,2}$, W. Collmar ${ }^{1}$, and V. Schönfelder ${ }^{1}$ \\ 1 Max-Planck-Institut für extraterrestrische Physik, PO Box 1603, 85740 Garching, Germany \\ 2 High Energy Astrophysics Lab, Institute of High Energy Physics, PO Box 918-3, Beijing 100039, PR China
}

Received 13 May 2002 / Accepted 1 October 2002

\begin{abstract}
We report the detection of an unidentified $\gamma$-ray source near the Galactic plane by the COMPTEL experiment aboard the Compton Gamma-Ray Observatory. The source is detected at a significance level of $\sim 7.2 \sigma$ in the energy range $1-3 \mathrm{MeV}$ and at $\sim 4.6 \sigma$ in the lower $0.75-1 \mathrm{MeV}$ band in the time period March to July 1995 . At energies above $3 \mathrm{MeV}$ are only marginal hints or upper limits obtained. The $\mathrm{MeV}$ spectrum has a soft shape. Strong flux variability is found within one year at energies below $3 \mathrm{MeV}$. Possible counterparts of galactic and extragalactic nature are discussed.
\end{abstract}

Key words. $\gamma$ rays: observations

\section{Introduction}

The Compton Gamma-Ray Observatory (CGRO), carrying $4 \gamma$-ray experiments (BATSE, OSSE, EGRET, COMPTEL), has observed the universe in $\gamma$-ray energies with unprecedented sensitivity between its launch in April 1991 and its reentry into the earth atmosphere in June 2000. These observations provided many new and exciting results, particularly the detection of many $\gamma$-ray point sources. Surprisingly, a big fraction of them is still unidentified. Among the 271 EGRET $\gamma$-ray sources listed in the 3rd EGRET catalogue for energies above $100 \mathrm{MeV}$, 171 are unidentified (Hartman et al. 1999). Some of them, located at low latitudes $\left(|b|<10^{\circ}\right)$, show a strong positional correlation with SNRs and OB associations (e.g. Romero et al. 1999), while some others $\left(|b|<10^{\circ}\right)$ do not coincide with any known potential $\gamma$-ray emitters (Torres et al. 2001a). This absence of positional coincidences might indicate the presence of a new class of $\gamma$-ray sources (Torres et al. 2001a).

COMPTEL was sensitive to soft $\gamma$-rays $(0.75-30 \mathrm{MeV})$ and finally opened the MeV band - which basically was unknown prior to CGRO - as a new astronomical window. Apart from $\gamma$-ray bursts, unidentified sources and AGN are the majority of the COMPTEL detections. While the sum of all others (pulsars, stellar black-hole candidates, supernova remnants and $\gamma$-ray line sources) are about 12, the first COMPTEL catalogue lists 10 AGN and 9 unidentified $\gamma$-ray sources (Schönfelder et al. 2000). In this paper we report the detection of a further unidentified COMPTEL source, which shows a variable MeV flux and is located in the galactic plane.

Send offprint requests to: S. Zhang, e-mail: szhang@mail.ihep.ac.cn

\section{Instrument and data analysis}

The imaging Compton Telescope COMPTEL is sensitive to $\gamma$-rays in the energy range $0.75-30 \mathrm{MeV}$ with energydependent energy and angular resolution of 5\%-8\% (FWHM) and $1.7^{\circ}-4.4^{\circ}(F W H M)$, respectively. Imaging in its large ( $\sim 1$ steradian) field of view is possible with a location accuracy of the order of $1^{\circ}-3^{\circ}$ depending on source flux. The effective area of COMPTEL drops with increasing offset to the pointing direction. At an offset angle of $\sim 30^{\circ}$ it is decreased to $\sim 50 \%$ (energy dependent). For details on the COMPTEL experiment see Schönfelder et al. (1993).

Skymaps and source parameters, like detection significances, fluxes and flux errors, can be obtained via the maximum likelihood method, which is implemented in the standard COMPTEL data analysis package. The detection significance is derived from the quantity $-2 \ln \lambda$, where $\lambda$ is the ratio of the likelihood $L_{0}$ (background) and the likelihood $L_{1}$ (source + background). The quantity $-2 \ln \lambda$ has a $\chi_{3}^{2}$ (3 degrees of freedom) distribution for a source search and a $\chi_{1}^{2}$ distribution for a flux estimate of a known source (de Boer et al. 1992). An estimate for the instrumental background of COMPTEL is derived by using the standard filter technique in the COMPTEL data space (Bloemen et al. 1994). In the presented analysis we applied instrumental point spread functions assuming an $E^{-2}$ power law shape for the source spectrum. The nearby known COMPTEL source, the $\gamma$-ray pulsar PSR 1509$58\left(l / b=320.3^{\circ},-1.2^{\circ}\right)$, has been included in the analyses, i.e. its fluxes were estimated simultaneously with the ones of the new source by a fitting process, and have been subtracted off in the maps of Fig. 1. The same procedure was applied for the 
celestial background components, the galactic and extragalactic diffuse gamma-ray radiation.

\section{Observations}

CGRO observations were organized in so-called CGRO "Mission Phases" and "Viewing Periods" (VPs). A "Phase" covers typically 1 year and contains many VPs, which typically last for 1 to 2 weeks (see e.g. Table 1). We have analyzed 48 such VPs, which are selected to be between the beginning of the CGRO mission in 1991 and the second reboost of CGRO in March 1997 (within CGRO Phase 6), and which have pointing offsets between instrument and $\gamma$-ray source $\left(l / b=311.5^{\circ}\right.$, $\left.-2.5^{\circ}\right)$ of less than 50 degrees. These observations are located in the first five CGRO Phases and sum up to an effective exposure (100\% COMPTEL directly on target) of 35.26 days on the source position.

\section{Results}

\subsection{Detections}

Evidence for the $\mathrm{MeV}$ source was first found by analyzing CGRO VP 424 (July 10-25, 1995) where the radio galaxy Centaurus A was the prime CGRO target. In the 1-3 MeV band a bright source with a detection significance of $5.2 \sigma$ is visible at a position near $l / b=311.5^{\circ},-2.5^{\circ}$. In addition, hints for this source were found in each of the 6 previous VPs covering this sky region. By combining these 7 VPs, which cover the time period March 21 to July 25 in 1995 (Table 1), we derive the best detection significances of $7.2 \sigma$ in the $1-3 \mathrm{MeV}$ band and of $4.6 \sigma$ in the $0.75-1 \mathrm{MeV}$ band by assuming 3 degrees of freedom (Fig. 1). The probability of detecting randomly an unknown source with a $-2 \ln \lambda$ value of 60 (corresponding to $7.2 \sigma$ ) in the COMPTEL $1-3 \mathrm{MeV}$ band is $5.4 \times 10^{-10}$ by taking into account the trials for searching in all individual VPs in four energy bands from the beginning of CGRO mission in 1991 to its second reboost in 1997 . Above $3 \mathrm{MeV}$ the evidence for the source becomes marginal. The source fluxes in the 7 VPs are roughly the same (Fig. 2). The detection significance is largest in VP 424 due to the best exposure (Table 1). The derived source fluxes are given in Table 2. By combining the observations of the first five CGRO Phases, we derive timeaveraged significances at energies below $3 \mathrm{MeV}$ ( 3 d.o.f.) of $4.7 \sigma$ in the $0.75-1 \mathrm{MeV}$ band, and $3.1 \sigma$ in the $1-3 \mathrm{MeV}$ band. Again, at higher energies only hints are obtained (see Table 2).

The most likely source location, the peak of maximum of likelihood ratio distribution in the $1-3 \mathrm{MeV}$ band of the combination of 7 VPs in 1995 , is at $l / b=311.5^{\circ},-2.5^{\circ}$. The error location contours are drawn from this distribution (see Fig. 5).

\subsection{Variability}

To check for possible source variability, we fit the fluxes of the individual VPs of the first five CGRO Phases in the 4 standard bands with a constant flux. In the $1-3 \mathrm{MeV}$ band is a $\chi^{2}$ value of 94.6 derived, which corresponds to a significance of $4.1 \sigma$ that the source is variable, and - subsequently - to a probability
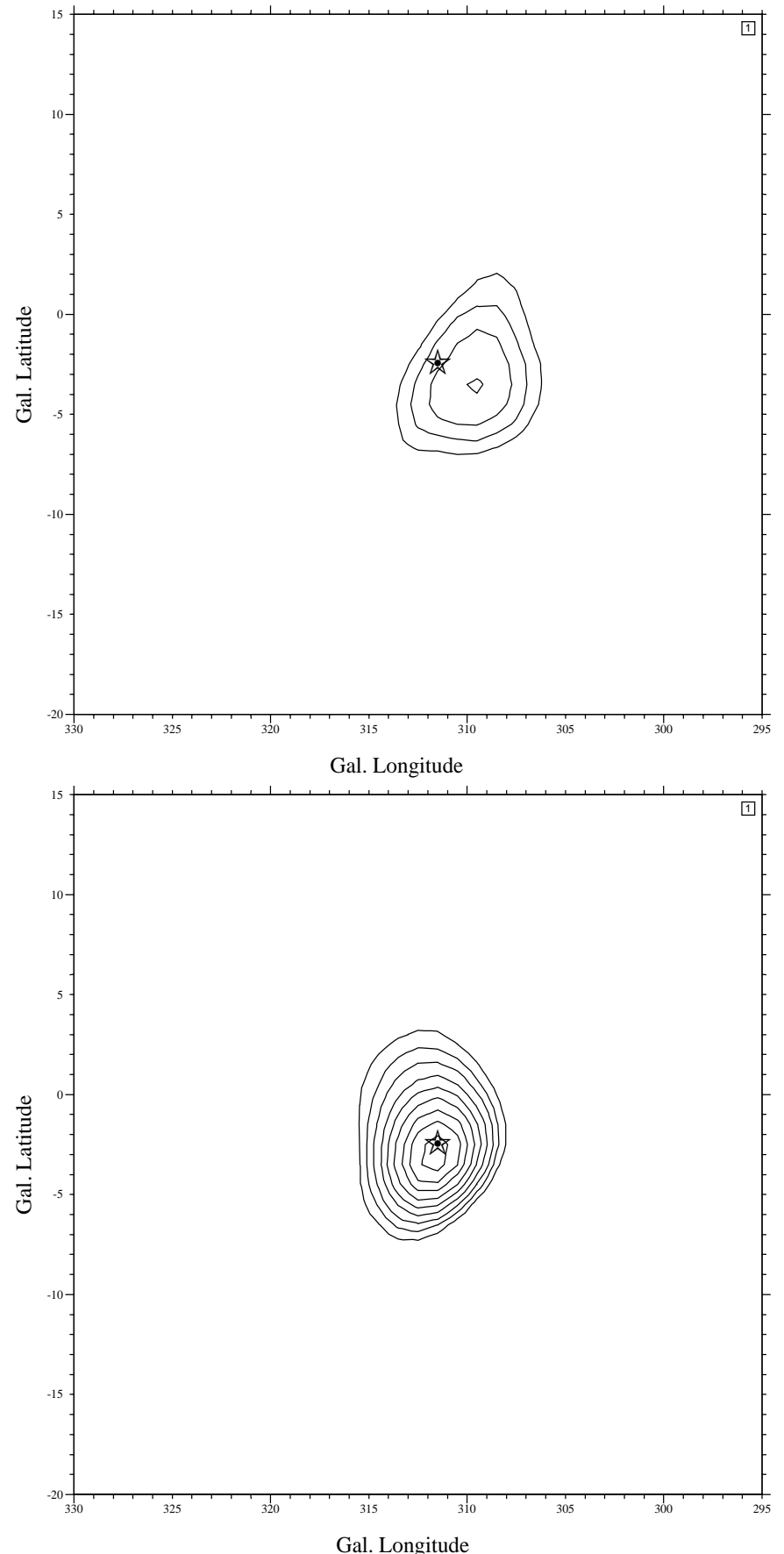

Fig. 1. Maps of the sky region of the unknown $\mathrm{MeV}$ source in the $0.75-1 \mathrm{MeV}$ band (top) and the 1-3 MeV band (bottom) for VPs 414-424. The star $(\star)$ represents the most likely source position. The known MeV source PSR 1509-58 as well as the diffuse $\gamma$-ray emission are subtracted off from the maps. The contour lines start at a detection significance level of $3 \sigma$ with steps of $0.5 \sigma$.

of $\sim 4.8 \times 10^{-5}$ that the source is constant. The flux variation is not significant in the other three energy bands $(<3 \sigma)$. Applying the same procedure to the light curves of the first five CGRO Phases (Fig. 3), i.e. averaging over relevant VPs, we derive a similar result: significant $(3.9 \sigma)$ flux variability in the $1-3 \mathrm{MeV}$ band, and insignificant one in the others. 
Table 1. COMPTEL observations of the sky region of the unidentified $\gamma$-ray source during its high state in 1995 (CGRO Phase 4). The CGRO VPs, their time periods in calendar date and TJD, prime observational targets of CGRO, pointing offset angles and effective exposures are given.

\begin{tabular}{cccccc}
\hline \hline VP & $\begin{array}{c}\text { Date } \\
(\mathrm{dd} / \mathrm{mm} / \mathrm{yy})\end{array}$ & TJD & Object & Offset angle & $\begin{array}{c}\text { Effective exposure } \\
\text { days }\end{array}$ \\
\hline 414.0 & $21 / 03 / 95-29 / 03 / 95$ & $9797-9805$ & Vela Pulsar & $33^{\circ}$ & 0.55 \\
414.3 & $29 / 03 / 95-04 / 04 / 95$ & $9805-9811$ & GRO J1655-40 & $35^{\circ}$ & 0.58 \\
415.0 & $11 / 04 / 95-25 / 04 / 95$ & $9818-9832$ & LMC & $42^{\circ}$ & 0.67 \\
421.0 & $06 / 06 / 95-13 / 06 / 95$ & $9874-9881$ & Gal. Center & $43^{\circ}$ & 0.28 \\
422.0 & $13 / 06 / 95-20 / 06 / 95$ & $9881-9888$ & Gal. Center & $43^{\circ}$ & 0.27 \\
423.5 & $30 / 06 / 95-10 / 07 / 95$ & $9898-9908$ & PKS 1622-297 & $36^{\circ}$ & 0.70 \\
424.0 & $10 / 07 / 95-25 / 07 / 95$ & $9908-9923$ & Cen A & $20^{\circ}$ & 2.39 \\
\hline
\end{tabular}

Table 2. Fluxes of the unknown $\gamma$-ray source, the time periods and the effective exposures for different observational combinations are listed. No source observation in CGRO Phase 6 before the second reboost of CGRO is carried out. The error bars are $1 \sigma$ and the upper limits are $2 \sigma$.

\begin{tabular}{ccccccc}
\hline \hline Period & TJD & Effective exposure & \multicolumn{4}{c}{ Flux $\left(10^{-5} \mathrm{ph} \mathrm{cm}^{-2} \mathrm{~s}^{-1}\right)$} \\
& & days & $0.75-1 \mathrm{MeV}$ & $1-3 \mathrm{MeV}$ & $3-10 \mathrm{MeV}$ & $10-30 \mathrm{MeV}$ \\
\hline Phase 1 & $8392-8943$ & 9.29 & $6.0 \pm 3.1$ & $<6.0$ & $1.7 \pm 1.6$ & $0.8 \pm 0.6$ \\
Phase 2 & $8943-9216$ & 5.87 & $4.2 \pm 3.5$ & $<10.1$ & $<3.9$ & $1.8 \pm 0.9$ \\
Phase 3 & $9216-9629$ & 7.45 & $<12.5$ & $6.7 \pm 3.8$ & $2.2 \pm 1.6$ & $<1.6$ \\
Phase 4 & $9629-9993$ & 8.42 & $13.7 \pm 3.8$ & $21.2 \pm 3.5$ & $4.6 \pm 1.6$ & $<1.2$ \\
Phase 5 & $9993-10371$ & 4.23 & $18.6 \pm 4.7$ & $<14.7$ & $<4.8$ & $<2.6$ \\
VPs 414-424 & $9797-9923$ & 5.44 & $19.9 \pm 4.6$ & $36.6 \pm 4.7$ & $5.4 \pm 2.3$ & $<1.8$ \\
Phases 1-5 & $8392-10371$ & 35.26 & $8.4 \pm 1.7$ & $6.1 \pm 1.6$ & $1.6 \pm 0.8$ & $0.5 \pm 0.3$ \\
\hline
\end{tabular}

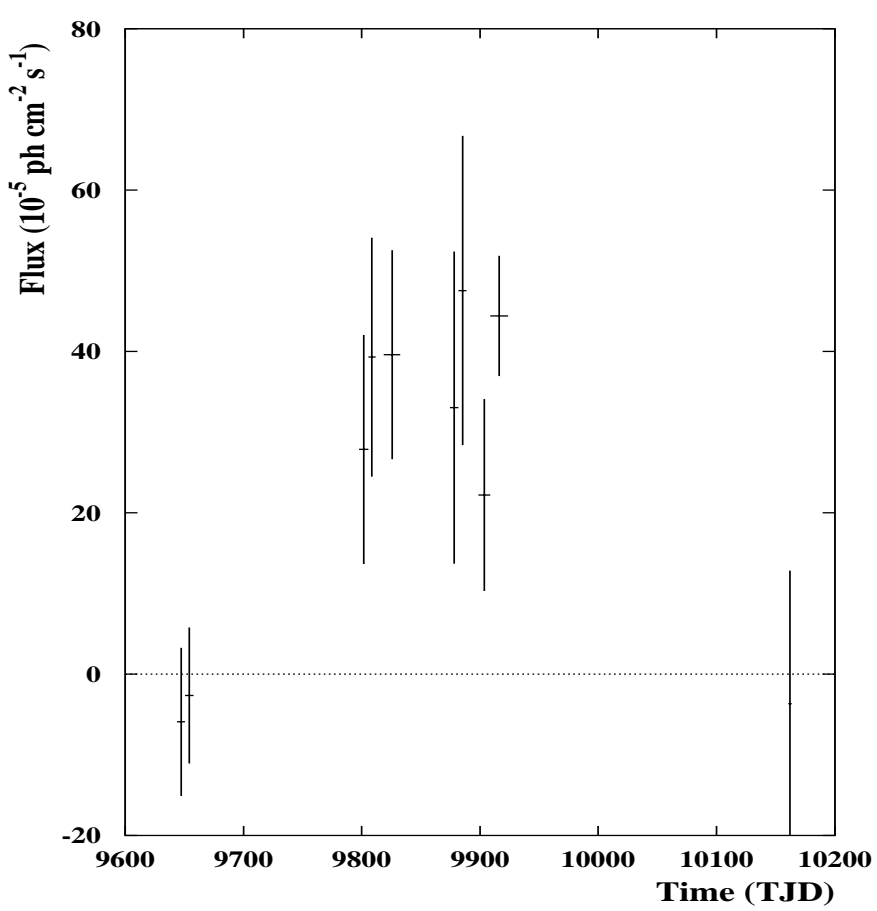

Fig. 2. 1-3 MeV light curve of the unknown $\gamma$-ray source between October 1994-March 1996. This period covers the outburst. Each bin represents an individual CGRO VP. The error bars are $1 \sigma$.

As was pointed out the $\chi^{2}$ statistics is statistical only and therefore does not account for any systematic errors which might be inherent in the data (e.g. instrumental trends). To overcome this uncertainty it was suggested (e.g. Zhang et al. 2000; Torres et al. 2001a) to normalize the measured variability to the measured variability of sources, which are considered to be

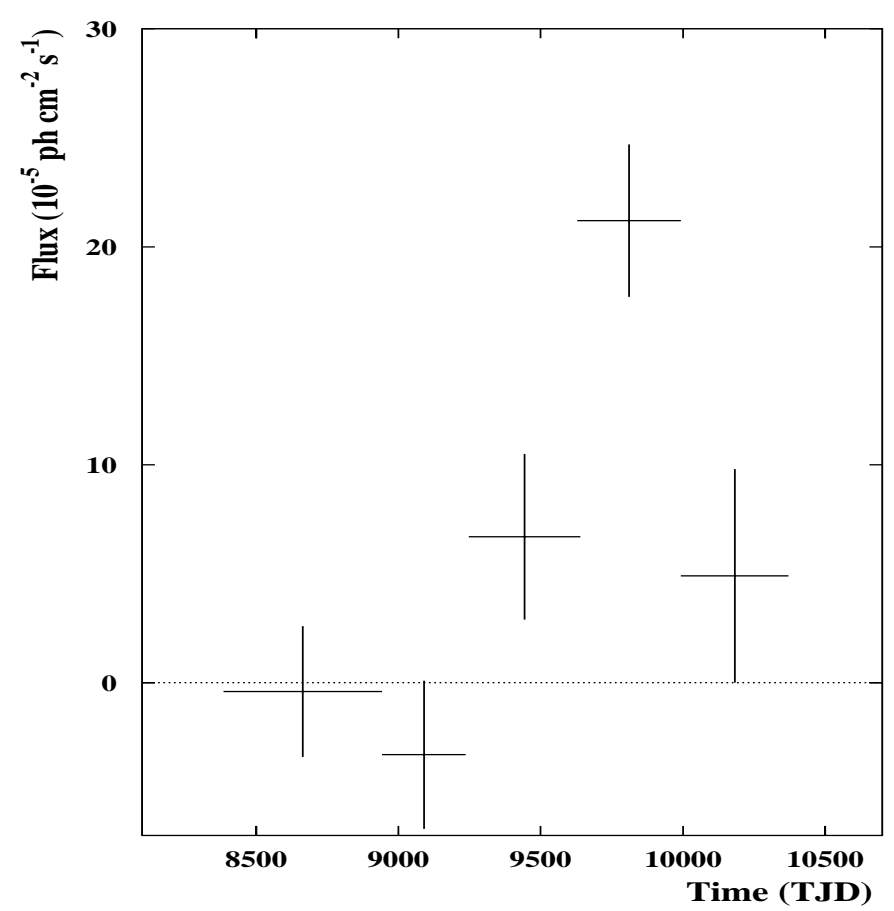

Fig. 3. Light curve of the unknown $\gamma$-ray source in the $1-3 \mathrm{MeV}$ band. Each bin is averaged over an individual CGRO Phase up to Phase 5. The error bars are $1 \sigma$.

non-variable. To do so, we calculated the so-called $I$ variability index (for definition and details see Zhang et al. 2000, and Torres et al. 2001a)

$I=\frac{\mu_{\text {source }}}{\mu_{\text {ref. sources }}}$ 
which establishes how variable a source is with respect to a given source population. Using the data of the first 5 CGRO Phases (Fig. 3) and compare them to the same type of data for the prominent and rather stable $\mathrm{MeV}$ sources Crab (pulsar + nebula), Cyg X-1, and 3C 273 (e.g. Collmar et al. 1999), we derive an $I$ index of 5.2 in the $1-3 \mathrm{MeV}$ band, which is just above the $3 \sigma$-level for variability (Torres et al. 2001b). Because Crab is the only significantly detected pulsar in this band, we added the X-ray binary Cyg X-1 and the quasar 3C 273 to the reference sample. These two source types are a priori not considered to be constant in $\gamma$-ray flux, which - in fact - even strengthens the $I$ index given above.

The highest source fluxes are obtained in CGRO Phase 4. Figure 2 shows the $1-3 \mathrm{MeV}$ light curve covering this period. There are 2 VPs, 402 and 402.5 (October 18-November 1, 1994), prior to the combination of the 7 VPs where the source was most prominent. The source is not detected in these 2 VPs, which proves variability on a time scale of less than 1 year, because the source rose from a non- to $7.2 \sigma$-detection in the 1-3 MeV band. The closest follow-up observation, VP 516.1 (March 18-21, 1996), by COMPTEL was carried out 8 months later. It provides a non-detection of the source in the $1-3 \mathrm{MeV}$ band (Fig. 2). The 1-3 MeV long-term (CGRO Phase 1 to 5) light curve shows that the source flux peaks overall in Phase 4 (Fig. 3).

\subsection{Energy spectra}

The energy spectrum of the source in VP 424 is consistent with a simple power-law shape of photon index $2.61_{-0.30}^{+0.33}$. For the combination of the 7 VPs (414-424) we also derive a soft spectrum of photon index $2.53_{-0.20}^{+0.23}$ (Fig. 4). The reduced $\chi^{2}$-values are 1.39 and 0.52 , respectively. The $1 \sigma$ errors are obtained by adding 1.0 to the minimum $\chi^{2}$-value. The spectra indicate that the source is weak at higher $\gamma$-ray energies (e.g. EGRET band), and - if no spectral turnover occurs - is bright at hard X-rays.

\section{Discussion}

Previously, no $\gamma$-ray source was known at the position of this $\mathrm{MeV}$ source. The closest known $\gamma$-ray-emitting object, the EGRET source 3EG J1410-6147, is located outside the $4 \sigma$ error region. This source coincides spatially with the COS B source 2CG 312-1 (Hermsen et al. 1977), and is tentatively identified with the supernova remnant G312.4-0.4 (Sturner \& Dermer 1995). To find possible counterparts, we searched the SIMBAD and NED databases for potential emitters of $\gamma$-rays near the source location. The obtained possible counterparts are overlaid on the COMPTEL error contours in Fig. 5.

There are $46 \mathrm{X}$-ray sources located within the $4 \sigma$ error location contour. With 2 exceptions, 2S 1417-624 and GS 1354-645, all of them are not identified at other wavelengths. 2S 1417-624 is a transient Be/X-ray binary pulsar system, with a Be star of $>5.9$ solar masses if assumes a neutron star of 1.4 solar masses. BATSE observed a huge outburst of this source at hard X-rays (pulsations up to $100 \mathrm{keV}$ were detected), which started on August 26, 1994 and lasted $~ 110$ days (Finger et al. 1996). COMPTEL observed the source twice

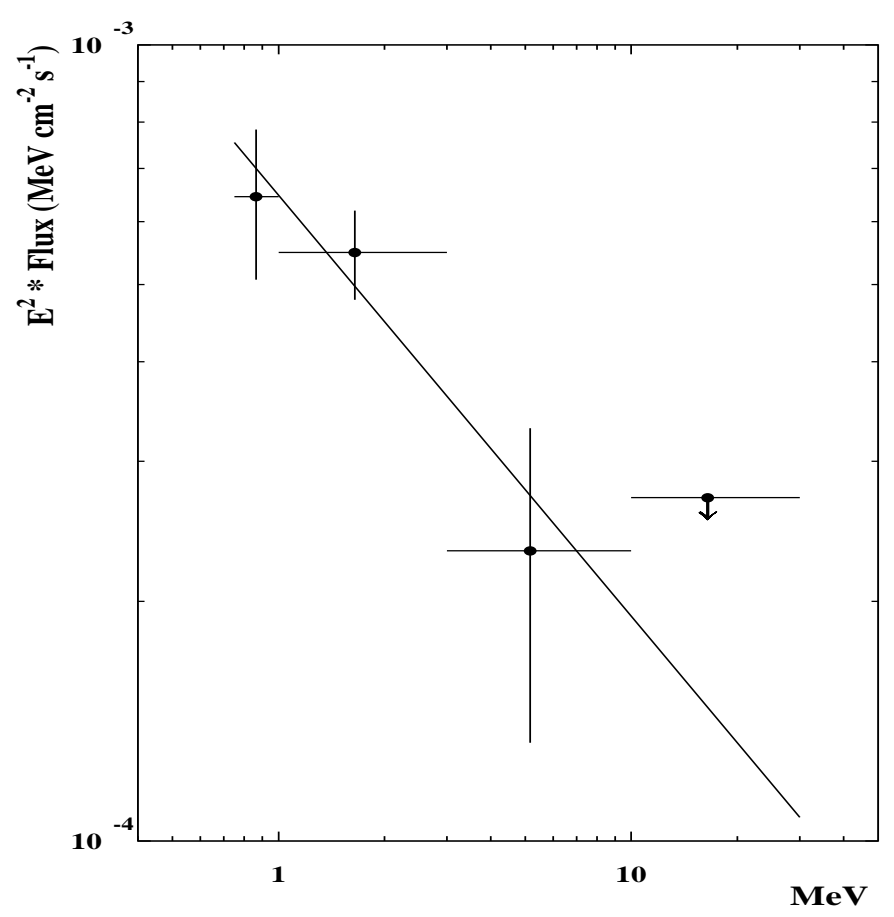

Fig. 4. The energy spectrum of the unknown $\gamma$-ray source for the combination of 7 VPs (414-424) in Phase 4. The solid line shows the bestfitting power law shape for the combination of VPs. The error bars are $1 \sigma$ and the upper limit is $2 \sigma$.

(VPs 402, 402.5) during this period but did not detect it. During the next $\sim 200$ days, 2 S 1417-624 showed 5 smaller outbursts (Finger et al. 1996), which coincide in time with the significant COMPTEL detection of the new MeV source in 1995. During the huge outburst in hard X-rays, the pulsed luminosity was found to be much less than it would be estimated from the spin-up rate. This implies that most of the power output is either unpulsed or outside of the hard X-ray range (Finger et al. 1996). Assuming 2S 1417-624 as counterpart of the MeV source, its $0.75-10 \mathrm{MeV}$ luminosity would be $2.5 \times 10^{37} \mathrm{erg} \mathrm{s}^{-1}$ by using its upper-limit distance of $11.1 \mathrm{kpc}$. This luminosity is comparable to the pulsed one of $\sim 2.2 \times 10^{37} \mathrm{erg} \mathrm{s}^{-1}$. 2S 1417 624 as counterpart would give an anticorrelation of the $\mathrm{X}$ - and $\gamma$-ray emission.

Cen X-3 is the only neutron star XRB pulsar system for which $\gamma$-ray emission above $1 \mathrm{MeV}$ was detected by CGRO. The significant detection of Cen X-3 by EGRET in October 1994 (Vestrand et al. 1997) proves that such objects could be occasional $\gamma$-ray emitters. In fact, Romero et al. (2001) have proposed the association of the $\gamma$-ray source $3 \mathrm{EG}$ $\mathrm{J} 0542+2610$ with the Be/X-ray transient A0535+26. They assume that the $\gamma$-rays are produced by the impact of relativistic protons (accelerated in the pulsar's magnetosphere) into the accretion disk. This, subsequently, generates $\gamma$-rays around $67.7 \mathrm{MeV}$ from $\pi_{0}$ decay, and also lower-energy $\gamma$-rays due to bremsstrahlung and Inverse-Compton scattering of relativistic secondary electron-positron pairs, which are produced by the decay of the charged pions. Because the source also generates a strong X-ray field, the high-energy $\gamma$-rays (EGRET band) could be absorbed, whereas the $\mathrm{MeV} \gamma$-rays could escape. 


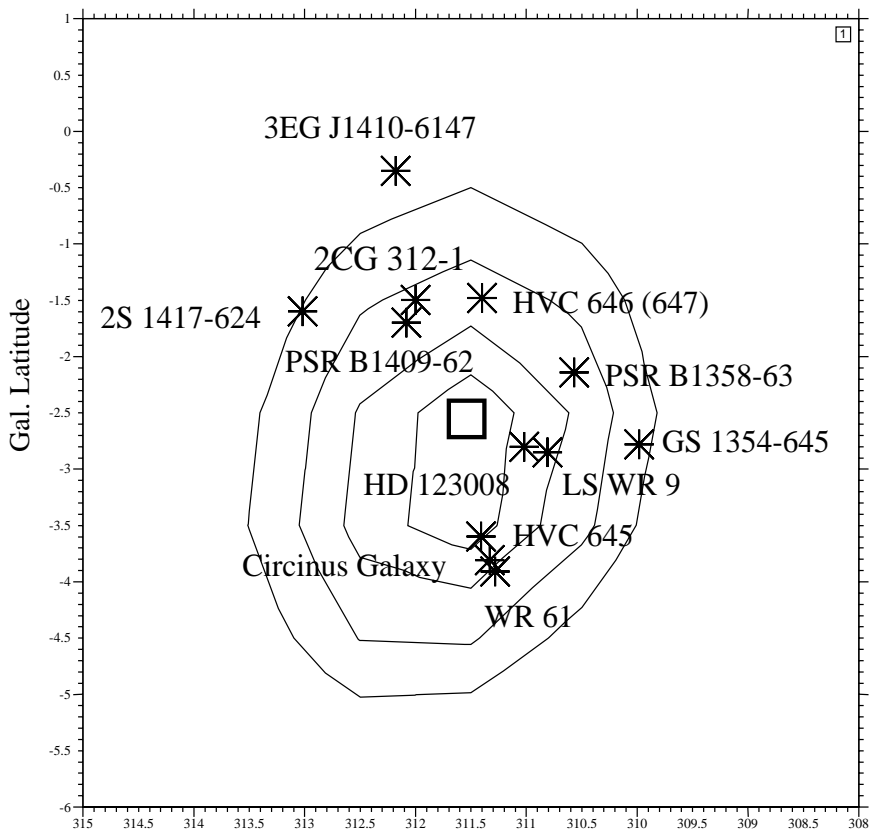

Gal. Longitude

Fig. 5. Error location contours for the unknown $\gamma$-ray source for the most significant detection: the $1-3 \mathrm{MeV}$ band for the combination of 7 VPs (414-424). The most likely source position ( $\square$ ) and possible counterparts are overlaid. The error contours start at $1 \sigma$ with steps of $1 \sigma$.

GS 1354-645 is a transient black-hole X-ray binary (XRB) system. MeV emission was detected by COMPTEL from 2 such systems: Cyg X-1, which shows a persistent $\mathrm{MeV}$ flux (e.g. McConnell et al. 2000), and GRO J0422+32, which showed a transient $\mathrm{MeV}$ flux and was detected only once at $\mathrm{MeV}$ energies, during a strong outburst in hard X-rays (van Dijk et al. 1995). However, no X-ray observations of GS 1354-624 during the activity period of the new COMPTEL source are reported. The MeV spectrum of the new source is soft (Fig. 4) and therefore is consistent in shape with the COMPTEL spectra of Cyg X-1 and GRO J0422+32.

A weak galactic microquasar or microblazar might also be the counterpart of this new MeV source. Recently Kaufman Bernadó et al. (2002) published a model for variable $\gamma$-ray emission from such objects. Relativistic electrons of a precessing jet upscatter stellar photons from the companion to $\gamma$-rays thereby generating a variable $\gamma$-ray emission. Although the model is tuned to $\mathrm{GeV} \gamma$-rays, a MeV source can be obtained by assuming a different spectrum for the relativistic electrons. Because the inverse-Compton emission is beamed but the disk emission is not, any of the unidentified (galactic) X-ray sources could - according to this scenario - be the counterpart. Due to different beaming factors in radio (synchrotron) and $\gamma$-rays, a strong radio source is not necessarily expected.

Massive stars, supernova remnants, high velocity clouds (HVC), and pulsars are also accelerating particles to relativistic energies by shocks or strong electromagnetic fields. Therefore such objects are also potential $\gamma$-ray emitters. Two HVCs are located within the $4 \sigma$ error contours. Gamma-ray emission from HVC was reported by Blom et al. (1997).
The time scale for flux variability of less than 1 year would provide constraints on the emission site in HVC. SIMBAD gives 3 massive stars, however no SNR within the $4 \sigma$ error contour. Two of them are Wolf-Rayet stars (WC9 for LSWR 9 Smith 1968; WN6 for WR 61 Torres-Dodgen \& Massey 1988), and the third one (HD 123008) is a O9.5I (Cruz-Gonzalez et al. 1974). Only HD 123008 has a photometric distance of $5.83 \mathrm{kpc}$ (Cruz-Gonzalez et al. 1974). There is no detection of non-thermal radio emission for these three massive stars. Two radio pulsars are located within the $3 \sigma$ error contour. They are not likely counterparts of the $\mathrm{MeV}$ source because the detected $\gamma$-ray pulsars do not show obvious time variability and typically have harder spectra (index $>-2.15$ ).

We investigated also extragalactic objects. There is only one AGN, the Circinus Galaxy, located within the $4 \sigma$ error contour. It is a Seyfert type 2 object at a distance of $\sim 3 \mathrm{Mpc}$. Seyfert galaxies were observed by CGRO, in particular by the OSSE experiment, to have a strong spectral cut off around $100 \mathrm{keV}$ (e.g. Johnson et al. 1997). Subsequently no Seyfert has yet been detected at $1 \mathrm{MeV}$ (e.g. Maisack et al. 1995) or above, which makes the Circinus AGN unlikely to be the counterpart. Torres et al. (2002) recently showed that by gravitational microlensing a distant $\gamma$-ray emitting blazar could be a strong and variable $\gamma$-ray source in the observer's frame without a radio or optical counterpart. According to their calculations is the magnification factor energy dependent, in the sense that it becomes stronger towards lower energies (their calculations covered the band between $100 \mathrm{MeV}$ and $10 \mathrm{GeV}$ ). This could mean, that a variable $\mathrm{MeV}$ source appears in the observer's frame without a visible $\mathrm{GeV}$ source. This scenario might provide a possibility to explain our transient $\mathrm{MeV}$ source by an extragalactic object although no obvious extragalactic object is within the error box.

$28 \mathrm{X}$-ray sources are embedded in the Circinus galaxy. Some of them are likely to be XRBs (Sambruna et al. 2001; Bauer et al. 2001). Placing the MeV source at this distance, a $0.75-10 \mathrm{MeV}$ luminosity of $\sim 1.8 \times 10^{42} \mathrm{erg} \mathrm{s}^{-1}$ is implied for the activity period in VPs 414-424. Assuming Eddington accretion, the black-hole mass in an XRB would have to be in excess of $\sim 10^{4} M_{\odot}$. Therefore, considering extragalactic XRBs in the nearby galaxy as the counterpart will result in a superluminosity problem, unless the source would be a microquasar or microblazar, for which jet emission is assumed (e.g. Markoff et al. 2001). Relativistic beaming could significantly enhance the apparent luminosity.

The MeV source resembles a special subgroup of unidentified EGRET sources located at low galactic latitudes $(|b|<$ $10^{\circ}$ ). These low-latitude sources show a highly variable flux, a soft $\gamma$-ray spectrum, and do not spatially coincide with any known potential $\gamma$-ray emitters (Torres et al. 2001a). Torres et al. (2001a) suggest that isolated Kerr-Newman black holes could be the objects behind. One of them, 3EG J1828+0142, was modeled by Punsly et al. (2000) based on that theory. Apart from time variability and soft spectrum, the model predicts in addition a broad spectral peak at $\mathrm{MeV}$ energies. Unfortunately, no further measurements, in particular at neighboring energies are available, to check whether the observed $\mathrm{MeV}$ emission is a peak or hump-like feature. 
In summary, searching the NED and SIMBAD databases provides no obvious counterpart within the $4 \sigma$ error contour. The source properties, flux variability and steep spectrum, resemble a special group of unidentified EGRET sources, which are also located near the galactic plane. However, XRB pulsars or microquasars or blazars could also generate such an emission profile.

\section{Conclusion}

A new COMPTEL $\gamma$-ray source is significantly detected at $\mathrm{MeV}$ energies between 0.75 and $3 \mathrm{MeV}$. The source is variable on a time scale of less than one year. The MeV spectrum for the high-state period is soft and can be represented by a simple power-law shape. Due to the absence of any simultaneous observations at other wavelengths, no identification is possible. Future contemporaneous $\mathrm{X}$ - and $\gamma$-ray observations have to be awaited to probe the nature of this source.

Acknowledgements. This research was supported by the German government through DLR grant 50 QV 9096 8, by NASA under contract NAS5-26645, and by The Netherlands Organization for Scientific Research NWO. This research has made use of the SIMBAD database operated at CDS, Strasbourg, France, and the NASA/IPAC Extragalactic Database (NED) which is operated by the Jet Propulsion Laboratory, California Institute of Technology, under contract with the National Aeronautics and Space Administration. The authors would like to thank the anonymous referee for the helpful comments.

\section{References}

Bauer, F. E., Brandt, W. N., Sambruna, R. M., et al. 2001, AJ, 122, 182

de Boer, H., Bennett, K., Bloemen, H., et al. 1992, in Data Analysis in Astronomy IV, ed. V. Di Gesù, L. Scarsi, R. Buccheri, et al. (New York: plenum Press), 241
Bloemen, H., Hermsen, W, Swanenburg, B. N., et al. 1994, ApJS, 92 , 419

Blom, J. J., Bloemen, H., Bykon, A. M., et al. 1997, A\&A, 321, 288

Collmar, W., Schönfelder, V., Strong, A. W., et al. 1999, Proc. of the 5th Compton Symp., AIP Conf. Proc., 510, 591

Cruz-Gonzalez, C., Recillas-Cruz, E., Costero, R., Peimbert, M., \& Torres-Peimbert, S. 1974, RMxAA, 1, 211

van Dijk, R., Bennett, K., Collmar, W., et al. 1995, A\&A, 296, L33

Finger, M. H., Wilson, R. B., \& Chakrabarty, D. 1996, A\&AS, 120, 209

Hartman, R. C., Bertsch, D. L., Bloom, S. D., et al. 1999, ApJS, 123, 79

Hermsen, W., Swanenburg, B. N., Bignami, G. F., et al. 1977, Nature, 269, 494

Johnson, W. N., Zdziarski, A. A., Madejski, G. M., et al. 1997, in Proc. of the 4th Compton Symp., AIP Conf. Proc., 410, 283

Kaufman Bernadó, M. M., Romero, G. E., \& Mirabel, I. F. 2002, A\&A, 385, L10

Maisack, M., Collmar, W., Barr, P., et al. 1995, A\&A, 298, 400

Markoff, S., Falcke, H., \& Fender, R. 2001, A\&A, 372, L25

McConnell, M., Ryan, L., Collmar, W., et al. 2000, ApJ, 543, 928

Punsly, B., Romero, G. E., Torres, D. F., \& Combi, J. A. 2000, A\&A, 364,552

Romero, G. E., Benaglia, P., \& Torres, D. F. 1999, A\&A, 348, 868

Romero, G. E., Kaufman Bernadó, P., Combi, J. A., \& Torres, D. F. 2001, A\&A, 376, 599

Sambruna, R. M., Brandt, W. N., \& Chartas, G. 2001, ApJ, 546, L9

Schönfelder, V., Aarts, H., Bennett, K., et al. 1993, ApJS, 86, 657

Schönfelder, V., Bennett, K., Blom, J. J., et al. 2000, A\&AS, 143, 145

Sturner, S. J., \& Dermer, C. D. 1995, A\&A, 293, L17

Smith, F. 1968, MNRAS, 138, 109

Torres-Dodgen, A. V., \& Massey, P. 1988, AJ, 96, 1076

Torres, D. F., Romero, G. E., Combi, J. A., et al. 2001a, A\&A, 370, 468

Torres, D. F., Pessah, M. E., \& Romero, G. E. 2001b, Astron. Nachr., 322,223

Torres, D. F., Romero, G. E., \& Eiroa, E. F. 2002, ApJ, 569, 600

Vestrand, W. T., Srekumar, P., \& Mori, M. 1997, ApJ, 483, L49

Zhang, L., Zhang, Y. J., \& Cheng, K. S. 2000, A\&A, 357, 957 